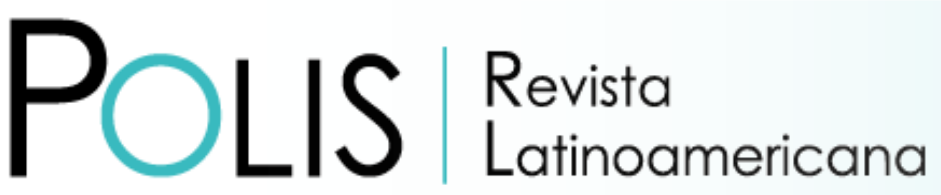

V20 | N58 | 2021

DOI: $10.32735 /$ S0718-6568/2021-N58-1577

\title{
La economía como argumento constitucional. El caso de la descentralización fiscal en Colombia
}

\author{
Alejandro Angel \\ Universidade Federal de Santa Catarina. Florianópolis, Brasil. \\ Email: alejandro.angel@posgrad.ufsc.br
}

Recibido: 28.08 .2020 | Aceptado: 02.12.2020

\begin{abstract}
Resumen: La Constitución de 1991 estableció que Colombia se organizaba en forma de República unitaria, descentralizada y con autonomía de sus entidades territoriales. Esto estaba en línea con las olas de democratización y las recomendaciones de la literatura sobre federalismo fiscal. Diez años después de promulgada la constitución, en un momento de profunda crisis económica y fiscal, hubo una reforma sustancial al esquema de transferencias que debía hacer el gobierno central a los gobiernos subnacionales, que le permitió al primero recuperar buena parte de los recursos fiscales que habían sido destinados a los gobiernos territoriales. Se argumenta aquí que, de la misma forma que las constituciones en América Latina se mueven al vaivén de las coaliciones partidarias, la constitución colombiana se cambió al vaivén de la economía. Esto le permitió al gobierno central retomar los recursos que había cedido por las reformas constitucionales sin necesariamente resolver definitivamente el déficit fiscal.
\end{abstract}

Palabras clave: Descentralización; crisis económica; cambios constitucionales; transferencias; política fiscal.

\section{The economy as constitutional argument. The case of fiscal decentralization in Colombia}

\begin{abstract}
The Colombian Constitution of 1991 stated that the country was organized as a unitarian decentralized republic with autonomous territories. This matched the waves of democratization and also followed the recommendations of the literature on fiscal federalism. Ten years after enacting the constitution, in a moment of deep economic and fiscal crises, a thorough reform to the intergovernmental transfers through which the central government financed subnational governments was enacted. This allowed the central government to recover a considerable portion of the fiscal resources that had previously ceded to subnational governments. It is argued that, in the same way that Latin American constitutions change depending on the reconfiguration of partisan coalitions, the Colombian constitution changed at the pace of economic performance. In consequence, the central government recovered the fiscal resources previously transferred due to constitutional reforms without necessarily solving its fiscal deficit.
\end{abstract}

Keywords: Decentralization; economic crisis; constitutional changes; transfers; fiscal policy. 


\section{A economia como argumento constitucional. O caso da descentralização fiscal na Colômbia}

Resumo: A Constituição colombiana de 1991 estabeleceu que o país se organizava em forma de república unitária, descentralizada e com entes territoriais autônomos. Isso seguia as linhas das ondas de democratização e das recomendações da literatura sobre o federalismo fiscal. Dez anos depois de ter sido promulgada a constituição, em um momento de profunda crise econômica e fiscal, houve uma reforma considerável ao esquema de transferências que o governo central devia fazer aos governos subnacionais, permitindo assim ao primeiro recuperar parte importante dos recursos fiscais que tinham sido alocados aos governos territoriais. Se argumenta que da mesma forma que as constituições na América Latina mudam segundo a configuração das coalizões partidárias, a constituição colombiana mudou segundo as condições da economia. Isto permitiu ao governo central recuperar os recursos fiscais que tinha transferido previamente devido às disposições constitucionais, sem por isso resolver definitivamente o problema do déficit fiscal.

Palavras-chave: Descentralização; crise econômica; mudanças constitucionais; transferências; política fiscal.

\section{Como citar este artículo:}

Angel, A. (2021). La economía como argumento constitucional. El caso de la descentralización fiscal en Colombia. Polis Revista Latinoamericana, 20 (58), 43-57 doi: http://dx.doi. org/10.32735/S0718-6568/2021-N58-1577

\section{Introducción}

Las intenciones de cambio social que desembocan en procesos de cambio constitucional, se refieren a una multiplicidad de dimensiones de la vida en sociedad que posteriormente se ven reflejados, en grados variables, en el texto constitucional que resulta de dichos procesos. Esa confluencia de factores lleva a que cambios institucionales vayan de la mano con procesos de democratización y liberalización económica tal y como se observó en varios procesos de cambio institucional tanto en América Latina como en Europa del Este entre los años 1980s y 1990s (O'Donnell, 1993). Uno de los cambios institucionales que mejor reflejan la yuxtaposición de reivindicaciones sociales, que buscan ser concretadas en las nuevas constituciones, es la descentralización, tanto política, como económica. Inclusive, se entendió dicho proceso de descentralización como parte fundamental de los procesos de democratización y transición a la democracia debido a la centralización que tradicionalmente había sido implementada por gobiernos autoritarios (Jordana y Gomà, 2004, p. 11).'

Así, además de esta dimensión política, también existe una dimensión económica, i.e., la división de los recursos fiscales, cuya discusión en la literatura cuenta con dos grandes vertientes. Por un lado, se encuentran los estudios llamados de federalismo fiscal de segunda generación (Oates, 2005; Weingast, 2009, 2014), que, al contrario de aquellos de la llamada primera generación (Musgrave, 1959; Oates, 1972), incorporan variables políticas

1 Mardones Z (2006) analiza la relación entre democratización y descentralización para el caso chileno. 
e institucionales para el estudio de la división de recursos entre las esferas de gobierno de la manera más eficiente posible. Por el otro, se encuentran estudios que entienden el federalismo fiscal y la descentralización de recursos como el resultado de procesos políticos en los que diversos actores al interior de los estados-nación, buscan acceder y controlar ciertos recursos financieros como contraparte a las reivindicaciones de autonomía y a los arreglos políticos propios de cada país (Rezende y Oliveira, 2003, 2004; Théret, 2015).

Los procesos constituyentes, en algunos casos, tuvieron entre uno de sus objetivos consolidar y profundizar procesos de descentralización que venían manifestándose de tiempo atrás. En Colombia, el proceso constituyente fue el producto de un sinnúmero de factores políticos (Negretto, 2013, p. 166ff). Una de las disposiciones que más debate ha generado por sus implicaciones políticas, económicas y legales, fue la descentralización de recursos tributarios que buscó sustentar desde el punto de vista presupuestal la autonomía política a los diferentes territorios, tanto de los departamentos, cuanto de los municipios. A pesar del impacto que situaciones coyunturales tuvieron en la convocatoria y posterior labor de la asamblea constituyente, el proceso de descentralización fiscal venía desarrollándose por al menos veinte años (Junguito, Melo, y Misas, 1995). Sin embargo, en menos de una década y con una situación política diferente, el proceso de descentralización fiscal fue revertido con una reforma constitucional.

Si por un lado es claro que la coyuntura política es esencial para entender los continuos cambios constitucionales en América Latina en general, y en Colombia en particular, no es claro a priori la razón por la que se privilegiaron cambios al componente fiscal de la constitución, es decir, a la descentralización, sin cambiar también las otras dimensiones que acompañaron a la misma. En línea con los cuestionamientos sobre los posibles alcances e innovaciones en diversos ámbitos que han aportado las constituciones latinoamericanas (Gargarella, 2015), además de las realidades políticas que condicionan dichos procesos (Negretto, 2013), el presente trabajo se pregunta sobre los motivos de la reversión de la descentralización fiscal en Colombia, sucedida a principios de siglo. La hipótesis que se presenta es que la coyuntura económica de finales de la década de 1990 en Colombia, marcada por una crisis pronunciada, justificó el cambio en el texto constitucional concerniente a la descentralización fiscal. Así como los equilibrios partidarios son importantes para entender los diseños institucionales y sus posibles alcances, la coyuntura económica también es importante para entender las posibilidades de cambio perenne que las constituciones pueden aportar en América Latina.

El presente trabajo está dividido en cuatro secciones incluyendo la presente introducción. Las dos secciones siguientes presentan, por una parte, los antecedentes del proceso de descentralización fiscal que se consolidó en 1991 en Colombia y, por la otra, las condiciones económicas y fiscales a lo largo de la década de 1990 que culminaron en el año 2001 con la reforma constitucional de las transferencias, respectivamente. Una última sección discute el tema y presenta el producto de estas reflexiones. 


\section{Antecedentes fiscales de la constituyente de 1991}

En 1968 fue implementado un cambio constitucional, a través del Congreso, que buscaba desconcentrar la acción estatal, principalmente en las políticas sociales, esto es, en educación y salud. El diagnóstico consistió en que la pérdida de importancia de los gobiernos subnacionales en la definición de las políticas públicas había llevado a una situación en la cual los indicadores sociales eran precarios. Un ejemplo emblemático de esto eran los problemas que tenían los departamentos para pagar los salarios de los funcionarios, principalmente profesores, quienes, por medio de movilizaciones y huelgas, lograron transferirle al gobierno central la responsabilidad de pagarle a los empleados de los departamentos, condicionando, de esta forma, el formato que la descentralización sostuvo durante buena parte de la segunda mitad del siglo XX (Acosta y Bird, 2005).

Dicha reforma constitucional también buscó modernizar la administración pública y en general el funcionamiento del Estado. No obstante, consideramos pertinente discutir el hecho de que una reforma administrativa, por más profunda que ella sea, deba hacerse mediante una modificación al texto constitucional de un país. Es cierto que la constitución vigente en ese momento había sido proclamada en 1886 y, por lo tanto, era razonable que casi un siglo después fuera necesario actualizar dicho texto para estar más acorde con las transformaciones que la sociedad colombiana había experimentado en ese lapso de tiempo. Sin embargo, otras reformas constitucionales habían sido implementadas previamente estableciendo parámetros de gobernabilidad y división de poder entre los partidos tradicionales lo cual pone de presente el papel "solucionador de problemas" que ha tenido el constitucionalismo en Colombia, dado que en repetidas ocasiones se han propuesto, e inclusive implementado, reformas constitucionales con el fin de solucionar problemas relativamente puntuales. Esto, como se discutirá más adelante para el caso de la descentralización, no necesariamente soluciona los problemas y conflictos que lanzaron dichos intentos de reforma. En cierto sentido, la tradición constitucionalista colombiana continúa ofreciendo reformas constitucionales como respuesta a conflictos de diversa índole (Uribe, 1985), sin resolverlos a cabalidad (Hurtado, 2006).

El caso específico de la división de los recursos tributarios entre los diferentes niveles de gobierno, no fue una excepción. Posterior a la reforma de 1968 se implementaron una serie de reformas que buscaban otorgar mayor autonomía financiera a los gobiernos subnacionales puesto que por décadas su participación en el reparto tributario había disminuido paulatinamente. Esto era un reflejo económico de la realidad política de un estado unitario en el que los gobiernos subnacionales tienen poca autonomía y funcionan como delegados del poder central en los respectivos territorios. Para el caso colombiano, los departamentos tenían en su órbita los impuestos a la cerveza, cigarrillo y licores en general, y vieron su participación en la carga tributaria total considerablemente disminuida, en parte debido a su estrecha base (Junguito y Rincón, 2004, p. 81). Con el propósito de 
subsanar dichos problemas se crearon una serie de transferencias intergubernamentales que disponían de una proporción creciente de los ingresos tributarios del gobierno central, inclusive de aquellos que habían sido recientemente creados con el fin de modernizar la administración tributaria y aumentar el recaudo (Bird, 1970).

De esta forma, los ingresos de los gobiernos subnacionales aumentaron, pero no llevaron a una mayor autonomía en el gasto, situación que no solucionó el problema inicial de la falta de oferta de servicios que había originado y justificado el proceso de descentralización. Desde el punto de vista estrictamente fiscal, sin embargo, la descentralización aparece como una cuestión de transferencia de recursos, directa o indirectamente, a pesar de que el proceso político implícito en la descentralización es la búsqueda por una mayor autonomía en la solución de los problemas particulares. En Colombia, no obstante, dicho proceso consistió en una simple desconcentración de la ejecución de las políticas sociales en el que las responsabilidades operativas en la ejecución de las políticas se localizan en el territorio mientras que las responsabilidades políticas continúan en manos de las autoridades centrales. Así, dada la continuidad de los problemas identificados a finales de la década de 1960, se creó la Misión de Finanzas Intergubernamentales (Colombia, 1981).

Esa misión criticó en su informe de 1981 el hecho de que las transferencias decretadas por las reformas de finales de la década de 1960 e inicio de la década de 1970 no tenían en cuenta adecuadamente las necesidades, capacidades y esfuerzo fiscal de cada una de las entidades territoriales. Así mismo propuso que fueran entregados a los gobiernos subnacionales recursos cada vez mayores a través de nuevos mecanismos y, fundamentalmente, que aquellos gobiernos, principalmente los de las ciudades más desarrolladas, se autofinanciaran hasta donde esto fuera posible, para permitir la liberación de recursos del gobierno central con destino a los otros gobiernos que no tienen la posibilidad de financiar sus propios gastos. Una vez más el sesgo fiscalista del proceso de descentralización quedó en evidencia al concentrarse fundamentalmente en el debate sobre la cantidad de recursos, sin que ello redundara necesariamente en mayor autonomía política o un mejoramiento visible de los servicios sociales.

Los años 1980 fueron un periodo conturbado en Colombia por cuenta de las múltiples problemáticas socio-políticas que aquel país atravesó (Jimeno, 2001). Desde el punto de vista estrictamente institucional, hubo algunas respuestas que intentaron atender algunas de las reivindicaciones que diversos sectores venían manifestando y a las que el Estado había respondido con una dosis considerable de represión, sin por ello caer necesariamente en los niveles observados en algunos regímenes burocrático-autoritarios. Un elemento importante de la respuesta institucional de ese periodo fue la reforma constitucional que permitía la elección directa de alcaldes. En efecto, es importante destacar que dicha reforma fue también una respuesta al proceso de paz que en ese momento se llevaba a cabo entre el gobierno de Colombia y las Fuerzas Armadas Revolucionarias de Colombia - FARC (Arenas, 
1985), que vieron en la descentralización y la elección de Alcaldes una posibilidad de nuevas prácticas políticas que permitirían la emergencia de nuevas fuerzas, inclusive aquellas venidas de la insurgencia, para permitir la apertura del sistema político (Restrepo, 2002, p. 524).

En esos años hubo una tensión permanente entre la necesidad de descentralizar, fuera por motivos político-administrativos ("Ios servicios serán mejor administrados si están cerca de los ciudadanos") o por motivos del orden fiscal ("es necesario descentralizar el gasto para liberar recursos del presupuesto") y el temor por parte de las autoridades centrales de que los gobiernos subnacionales no tuvieran las condiciones necesarias para ejecutar las políticas de forma idónea. Dicha tensión marcó ampliamente el proceso de descentralización en Colombia, convirtiéndola en un asunto recurrente. Así, era de esperar que, dado el fuerte centralismo presente durante muchos años - que inclusive permitió y articuló la consolidación del Estado-Nación - se mantuviera esa preocupación sobre la estabilidad, lo cual ejerció importantes presiones para mantener recursos y competencias, materializando dichas pretensiones a través de iniciativas ambiguas de transferencia a los gobiernos subnacionales. Dicha ambigüedad creó confusión en el proceso de política y, al mismo tiempo, legitimó esos temores en una suerte de profecía auto-cumplida.

Así, al llegar al momento constituyente, y más allá de analizar su importancia relativa respecto del conjunto de procesos que condujeron a la convocatoria de la Asamblea Nacional Constituyente (Ginsburg, Elkins, y Blount, 2009), era claro que muchos sectores sociales vieron en el mismo una salida plausible a los problemas que aquejaban a la sociedad colombiana. Por un lado, se concebía la reforma constitucional como una respuesta a la crisis institucional que vivía el Estado, pero también fue una oportunidad para reformular o profundizar reformas que venían de tiempo atrás. Las reformas institucionales de tipo liberal que instituyeron una amplia carta de derechos, entre otros avances, representaron un avance sustancial respecto de la tradición del país. En el ámbito de la descentralización territorial la constitución colombiana de 1991 representó tan sólo una profundización de una política que venía siendo implementada con antecedencia.

La próxima sección presenta la realidad de los gobiernos subnacionales colombianos después de haber recibido autonomía política y considerables recursos fiscales por parte del constituyente primario.

\section{La corta vida de la constitución de 1991}

La nueva constitución intentó reformar el sistema político colombiano al cambiar buena parte de las reglas de distribución del poder político y de recursos, promoviendo la inclusión de diversos sectores de la sociedad que hasta entonces se encontraban excluidos del propio sistema. En lo que se refiere a las relaciones intergubernamentales, la Constitución modificó las reglas que seguían esas relaciones, fundamentalmente porque los gobiernos subnacionales no dependían más del gobierno central, garantizándoles su autonomía. Sin 
embargo, esta renovada autonomía no necesariamente se tradujo en una distribución clara de las competencias que dichas entidades tenían para efectos de las políticas públicas. De tal forma, la constitución profundiza la crisis de los departamentos como estructuras de gobierno desprovistas de una función clara y específica, mientras que los municipios ejecutan las políticas públicas, tanto por cuestiones de proximidad a las comunidades, como por cuestiones de eficiencia económica. Por su parte, el gobierno central planifica y coordina, dejando a los departamentos sin un papel claro en el proceso de política.

En lo que se refiere a la distribución de recursos, el texto constitucional profundizó el proceso que ya estaba en curso en la década de 1980. El constituyente primario consideró que vincular el crecimiento de las transferencias intergubernamentales al crecimiento de los ingresos del gobierno central era la forma adecuada de evitar que el gobierno central tratara de coartar la recién adquirida autonomía. Sin embargo, dicha autonomía debe ser cualificada puesto que la misma no deriva de forma automática de la cantidad de recursos. De hecho, el constituyente limitó la autonomía de los gobiernos subnacionales, al condicionar las transferencias recibidas para determinados rubros: educación y salud. De esta forma, el mecanismo que aparentemente otorga a los gobiernos subnacionales mayor autonomía, en realidad condiciona y limita esta autonomía al vincular los nuevos ingresos venidos de las transferencias, a lo que el constituyente de forma centralizada define como la necesidad social y globalmente relevante. No obstante, eso no significa que el texto de la Constitución no tenga en cuenta las necesidades de la población colombiana. Inclusive, los críticos de la Constitución señalan que ella prometió mucho más allá de la capacidad del Estado colombiano de volverla realidad (Rodríguez, 2003, p. 3).

La constitución no definió propiamente la cantidad de recursos que le correspondería a cada nivel de gobierno, pero estableció que la cantidad de recursos debería estar de acuerdo con las necesidades de las poblaciones en los respectivos territorios. Poco después de concluido el proceso constituyente vinieron los desarrollos legales que indicarían la cantidad precisa de recursos que correspondería a los departamentos y municipios para que los mismos lo dedicaran a los sectores de educación y salud. ${ }^{2}$ Esto, cabe anotar, continuaba la tradición del proceso de descentralización en Colombia, en el que los gastos de políticas sociales jugaron un papel primordial como justificación y materialización del mismo. En este sentido, la constitución tan sólo dio continuidad a un proceso que ya se encontraba en marcha, tanto en lo político - al reconocer un grado de autonomía política que antes no existía -, cuanto en lo económico - con el aumento de los recursos a transferir (Perry y Rodríguez, 1991).

Esto, no obstante, fue rápidamente objeto de críticas y la conveniencia de dichos arreglos fue cuestionada desde diversos frentes. Una de dichas críticas se concentró en la posibili-

2 La Ley 60 de 1993 estableció dos fondos diferentes, ambos provenientes de los que recaudaba el gobierno central a través de los Ingresos Corrientes de la Nación. Por un lado, un renovado Situado Fiscal que debería incrementarse de $23 \%$ en 1994, lo cual ya representaba un aumento de ocho puntos porcentuales en la participación de un año para otro, hasta $24.5 \%$ en 1996. Por otro lado, la antigua Cesión de Ventas se transformó en las Participaciones Municipales, que deberían constituir 15\% de los Ingresos Corrientes de la Nación en 1994 y, a partir de este año, aumentar un punto porcentual hasta 22\% en 2001 (Acosta y Bird, 2005). 
dad que las crecientes transferencias a los gobiernos subnacionales iban a perjudicar el esfuerzo fiscal de los gobiernos, configurando así, la denominada pereza fiscal. Sin embargo, varios autores refutaron la hipótesis de pereza fiscal para los departamentos y municipios colombianos antes y después de la Constitución de 1991 (Cadena, 2002; Jaimes, 2020; Junguito et al., 1995). Adicionalmente, las principales críticas al proceso de descentralización se concentraron en las consecuencias de orden fiscal que tuvo para el gobierno central puesto que el déficit de este último aumentó a medida que aumentaron las transferencias a los gobiernos subnacionales (Junguito et al., 1995, p. 29), comenzando un largo debate sobre la importancia que tuvo el proceso de descentralización y su principal indicador económico, las transferencias constitucionales, en la crisis fiscal que enfrentó el gobierno central en la segunda mitad de la década de 1990.

Por otra parte, los gobiernos subnacionales muchas veces no supieron aprovechar los recursos que el constituyente les otorgó. Las transferencias fueron usadas por algunos gobiernos departamentales como garantía de préstamos obtenidos en el sistema financiero (Echavarria, Rentería, y Steiner, 2002, pp. 18-21; Sánchez, Olivera, y Cortes, 1997, p. 26). En la práctica, estos hipotecaron sus ingresos. Así, de la misma forma en que hipotecas pueden representar situaciones positivas o negativas, las deudas que contrajeron dichos gobiernos subnacionales fueron usadas tanto para realizar inversiones importantes como para financiar gastos más tradicionales (Clavijo, 2004, p. 155). Por su parte, la economía del país fue cayendo poco a poco en la más grave crisis desde la Gran Depresión, con crecimientos negativos del Producto Interno Bruto - PIB - de más de $4 \%$ y un déficit fiscal del gobierno central de cerca del 6\% del PIB (Lozano, 2001, pp. 1-2). Este fue el contexto económico que permitió el surgimiento del debate político acerca del papel que las transferencias - es decir, el proceso de descentralización - tuvieron sobre la economía colombiana de un modo general y sobre las finanzas del gobierno central en particular. Más allá del debate económico sobre el impacto que las transferencias intergubernamentales tuvieron en el deterioro de la posición fiscal del gobierno central de Colombia, aquí se pretende señalar la manera en la que dicho argumento fue utilizado para promover una reforma constitucional, a menos de una década de haber sido promulgado el texto original, que devolvió los recursos al gobierno central y, en efecto, retrocedió varias décadas en la discusión sobre el significado del proceso de descentralización.

En la práctica, esto muestra que la Asamblea Constituyente que promulgó en 1991 una nueva constitución en la que hubo importantes avances en multiplicidad de arenas -e.g., una generosa carta de derechos, el reconocimiento del carácter pluriétnico y multicultural de la nación colombiana, entre otras - no logró zanjar el debate político acerca del significado de la descentralización política y de recursos económicos en Colombia. De hecho, como fue señalado anteriormente, numerosos actores argumentaron que la razón del debilitamiento de las finanzas públicas colombianas a lo largo de la década de 1990 fueron las transferencias de recursos a los gobiernos subnacionales (Alesina, Carrasquilla, y Echavarria, 2000, p. 22; Clavijo, 2004, p. 148-149; Wiesner, 2004, p. 11). El propósito de este texto no es determinar la importancia relativa que tuvo el proceso de descentralización de recursos en 
la crisis fiscal del gobierno central de Colombia; no obstante, es importante señalar que la presencia de una crisis económica y las consecuencias que tuvo en las finanzas de todos los niveles de gobierno tuvieron un impacto considerable en la construcción de una coalición política suficientemente fuerte para alterar el texto constitucional original.

En nombre de la estabilidad macroeconómica se modificaron los esquemas de transferencias intergubernamentales que representaban un paso importante en la trayectoria de la descentralización colombiana (Iregui, Ramos, y Saavedra, 2001, p. 16; Sánchez, Smart, y Zapata, 2002, p. 8). Aunque el argumento fiscalista sobre el impacto que las transferencias tenían sobre las finanzas del gobierno central pueda tener una dosis de verdad, es importante señalar, por más obvio que pueda parecer, que las crisis económicas son eventos de carácter coyuntural mientras que el cambio propuesto para solucionar la crisis fiscal del gobierno central colombiano en su momento fue la alteración del texto constitucional que tiene por naturaleza un carácter permanente. Esto respalda la hipótesis que este texto defiende, es decir, de la misma forma que las prioridades electorales y políticas de los partidos y grupos políticos en América Latina determinan el compromiso que los mismos tienen frente a las instituciones creadas en el marco de la constitución (Negretto, 2013), las preocupaciones de orden fiscal que generó una crisis económica, fueron suficientes para promover un nuevo cambio en la distribución de recursos mediante una alteración en el texto constitucional.

Cabe, sin embargo, preguntarse si la definición de la cantidad de recursos que le corresponde a cada nivel de gobierno año tras año deba aparecer en un texto constitucional. Por otra parte, en ese mismo orden de ideas también es válido indagar si la política económica deba someterse a normas excesivamente rígidas que impidan perseguir los múltiples objetivos que la misma debe alcanzar. Lo cierto es que el vaivén que el proceso de descentralización fiscal presentó en Colombia a finales del siglo pasado, deja entrever que esa cuestión estaba lejos de ser un asunto resuelto, puesto que buena parte de los problemas que se generaron a raíz de la crisis bien pudieron haber sido objeto de desarrollos legales como de hecho muchos de ellos los fueron ${ }^{3}$ - que también podían presentar una solución aceptable, sin que por ello fuera necesario alterar el pacto político que se había construido alrededor de la descentralización de recursos. Adicionalmente, es importante destacar que en ningún momento existieron argumentos en el debate público colombiano, que pidieran cambios fundamentales al componente estrictamente político de la descentralización, es decir, la elección directa de las autoridades locales, tanto gobernadores departamentales como alcaldes en el caso de los municipios.

Es así como el debate político en el contexto colombiano fue reducido a mayor o menor descentralización fiscal dejando de lado las muchas dimensiones que intervienen en un

3 Mediante las leyes 549 y 550 de 1999 se les dio solución a los problemas de tipo pensional (jubilaciones) de los empleados departamentales, así como la posibilidad a los gobiernos departamentales de negociar con sus acreedores, respectivamente. Adicionalmente se creó un mecanismo de clasificación de los gobiernos departamentales según su capacidad de generación de ingresos, es decir, de pago de sus deudas, a modo de una regla fiscal (G. Restrepo y Alvarez, 2005). 
asunto tan complejo. Más allá de la conveniencia de un arreglo institucional más centralizado o más descentralizado, tanto en lo político, como fiscal, como de la ejecución de las políticas públicas (Prud'Homme y Shah, 2004), otras dimensiones se han visto relegadas o simplemente han sido ignoradas debido a visiones relativamente miopes del significado de dicho proceso de transformación que podría así mismo ofrecer respuestas a problemas más amplios que la simple distribución de recursos tributarios (D. Restrepo, 2004).

En la próxima sección se discutirán las consecuencias que la reforma constitucional de final de siglo, tuvo sobre el proceso de descentralización, con especial atención al papel que los municipios han jugado desde 1991 y la forma en la que la ausencia de crisis económica posterior al cambio constitucional de principios de siglo no revirtió a la situación original decidida por el constituyente primario.

\section{Discusión}

En los años siguientes a la reforma constitucional, las transferencias del gobierno central para los gobiernos subnacionales se desvincularon de los ingresos tributarios del primero y, como política compensatoria, dichas transferencias pasaron a vincularse al comportamiento de la inflación. La idea que la reforma que modificó las disposiciones constitucionales originales implantó es que el desempeño económico también es un argumento constitucional de peso y que, a la manera de los factores reales de poder característicos de la teoría constitucional moderna (Lassalle, 1946), este también debe ser considerado. Más allá de la discusión sobre si las rigideces presupuestales que se crearon al determinar el comportamiento de las transferencias según los ingresos tributarios del gobierno central y de los problemas de orden fiscal que esto pueda generar (Echeverry, Fergusson, y Querubín, 2004) fueron un desliz del constituyente primario o un intento de impedir retrocesos en el acceso a los servicios sociales, es menester pensar sobre la realidad que los gobiernos subnacionales afectados por dicha reforma tuvieron que enfrentar.

Para empezar, los recursos siguieron siendo distribuidos mediante complejas fórmulas. Si las disposiciones legales que desarrollaron la constitución usaban la proporción de habitantes con Necesidades Básicas Insatisfechas - NBI - además de otras medidas y porcentajes específicos, las reglas que las reemplazaron continuaron con esa estrategia. El financiamiento de la educación, pasa a ser distribuidos por alumno atendido, según un cálculo que realiza el gobierno central y que es multiplicado por la cantidad de alumnos en el sistema, según el nivel educativo y la región, además de la población por atender considerado como un porcentaje del valor por alumno atendido. Los recursos para la salud a su vez son destinados a realizar subsidios a la demanda (Sánchez, 2006, pp. 11-13). Así, de la misma forma que al inicio de la década de 1990, los instrumentos de implementación de las políticas sociales se adecuaron según el instrumento de focalización considerado más apropiado; de las NBI 
se pasó al conteo por estudiante, para el caso de la educación, mientras que la salud siguió el esquema vigente con la ayuda del Sistema de Selección de Beneficiarios - SISBEN - que venía funcionando previo a la reforma (Bottia, Cardona Sosa, y Medina, 2012).

Ante la pregunta ¿ेPara qué sirvió la asamblea constituyente de 1991 en Colombia?, la respuesta en lo que tiene que ver con la descentralización política es inequívoca: gracias a la constitución los gobiernos subnacionales lograron consolidarse como protagonistas de las políticas sociales que tienen mayor impacto en la vida de la población: salud, educación y agua potable y alcantarillado. Así lo quiso el constituyente y así se realizó. Ese protagonismo vino acompañado de autonomía económica en un primer momento, pero por razones políticas eso fue revertido, sin que necesariamente los servicios por los cuales dichos municipios eran responsables dejaron de funcionar, más allá de crisis puntuales en ciertas regiones. En lo que se refiere a la educación, es importante destacar que la cobertura aumentó en educación primaria y secundaria (Sánchez, 2006, pp. 21-30), pero la calidad medida por resultados en pruebas estándar aplicadas a todos los estudiantes presentó indicadores peores para la enseñanza pública (Melo, 2005, p. 25), situación que deja entrever el dilema que enfrentan las autoridades, cobertura o calidad. Esto debería dejar de lado el argumento centralista sobre la incapacidad de los gobiernos territoriales que inevitablemente deberían ser tutelados por los niveles de gobierno superiores, sean estos regionales o centrales. De hecho, la evidencia indica que los ingresos tributarios propios han sido razonablemente bien explotados por parte de los municipios y en menor medida por los departamentos (Sánchez, España, y Zenteno, 2015).

Por otra parte, como ya fue mencionado antes, la Asamblea Constituyente quiso representar al conjunto de la nación colombiana. Sin embargo, el conflicto armado que afecta a dicho país desde hace décadas no vio su fin con dicha asamblea a pesar de la inclusión de diversos grupos armados al margen de la ley que decidieron participar en dicho proceso político en su momento. Lamentablemente varios más no lo hicieron sin que por ello dejaran de usufructuar los cambios que la constitución estableció en lo que atañe a la distribución de los recursos que le corresponden, principalmente a los municipios, obedeciendo a la descentralización política y fiscal. Esta extraña situación se conoce como clientelismo armado puesto que grupos armados se comportan como grupos de presión en determinados territorios y hacen uso de los recursos que les corresponden a dichas administraciones por transferencias. Ese es un fenómeno que afecta a una multiplicidad de municipios, grandes y chicos, y es perpetrado por grupos irregulares de las más diversas índoles y corrientes ideológicas, que van cambiando de acuerdo tanto a las dinámicas del conflicto armado como del conjunto de actividades ilícitas que usan para financiarse (Rodríguez-Takeuchi, 2009; Rubio, 2002; Trejos Rosero, Guzmán, Badillo Sarmiento, y Martínez Martínez, 2020).

Por otra parte, en algunos casos, algunos autores se han preguntado sobre la utilidad del cambio constitucional estudiado aquí en términos estrictamente fiscales, decididamente, la razón que esgrimieron sus promotores para implementarla. La situación fiscal no cambió $y$, al contrario, la crisis se mantuvo haciendo que la trayectoria fiscal que el país presenta- 
ba en el año de 2003 fuera insostenible (Rincón, Berthel, y Gómez, 2003, p. 42), inclusive en el momento en que el nuevo esquema de las transferencias ya estaba vigente, dejando en evidencia que el déficit fiscal no era producto de las reformas descentralizadoras de la Constitución. ${ }^{4}$ Varios años después el déficit fiscal del gobierno central seguía siendo considerable, a pesar de no estar en los niveles críticos observados en los años más duros de la crisis económica que aquejó al país (ECLAC, 2019).

Los términos del debate que permitió el cambio constitucional, objeto de estudio aquí, fue una falsa dicotomía entre estabilidad macroeconómica y autonomía de los gobiernos subnacionales. Dichos términos fueron aceptados por todos los allí implicados, puesto que la dimensión estrictamente presupuestal y tributaria del proceso de descentralización, fue enfatizada también por aquellos que ahora disponían de la autonomía política que también había sido otorgada por la nueva constitución. La dimensión de la descentralización que debía tener un carácter de soporte a la autonomía política, acabó dominando el debate de fondo sobre la primera relegándola a un segundo plano. No obstante, los debates en torno al proceso de descentralización en Colombia, estaban lejos de haber sido resueltos una década después de la principal reforma constitucional en materia de repartición de recursos tributarios e, inclusive, también en materia de la responsabilidad en la provisión de los servicios sociales (Bird, 2012).

Por otra parte, más allá del complejo debate económico y constitucional en que se convirtió la descentralización, es crucial mirar con cierta perspectiva el camino recorrido puesto que el constituyente primario tenía como objetivo fundamental al profundizar el proceso de descentralización, crear los mecanismos suficientes para garantizar los derechos económicos, sociales y culturales en la forma de acceso a servicios de educación y salud principalmente. Aunque el conjunto de la población colombiana está lejos de contar con servicios ideales, es menester reconocer que ha habido transformaciones en los cerca de treinta años de vigencia de la constitución. Dicho esto, también es necesario reconocer que las reformas constitucionales no son todopoderosas y, por lo tanto, no pueden operacionalizar los derechos consagrados en sus textos. Su papel es justamente este, consagrar los derechos para que la sociedad haga lo necesario para garantizarlos, con lo cual las discusiones decenales sobre los cambios constitucionales necesarios para afrontar crisis políticas, económicas o sociales podrían pasar a darse cada cien años.

Tal y como se mostró en el presente trabajo, una crisis económica y la consecuente crisis fiscal que esta generó, consolidaron el argumento político para modificar la constitución de Colombia. Esto está en línea con lo planteado por Negretto (2013) sobre la inestabilidad de los arreglos constitucionales en América Latina, a pesar de que para el momento de la reforma aquí estudiada, la supervivencia del Estado colombiano también estaba siendo puesta a prueba, de la misma manera que una década atrás. Sin embargo, más allá de la diferencia en lo que respecta a los motivos constitucionales de Colombia, consideramos

4 Otros autores también señalaron que el déficit del gobierno central de Colombia era de carácter estructural y no obedecía exclusivamente a las transferencias. Para más detalles, ver Dillinger y Webb (1999) y Lozano y Aristizábal (2003). 
crucial señalar como punto central la importancia de la coyuntura, sea esta política, como en el análisis de Negretto, o bien sea económica como procuramos señalar aquí, en los asuntos constitucionales de América Latina pone de presente que las constituciones en América Latina son creaturas frágiles.

\section{Agradecimientos}

El autor agradece el financiamiento recibido por medio del Programa Nacional de Pós-doutoramento - PNPD de la Coordenação de Aperfeiçoamento para o Pessoal de Nível Superior - CAPES. Ministerio de Educación de Brasil.

\section{Bibliografía}

Acosta, O. L., y Bird, R. (2005). The Dilemma of Decentralization in Colombia. En R. Bird, J. M. Poterba, y J. Slemrod (Eds.), Fiscal Reform in Colombia: Problems and Prospects (pp. 247-286). Cambridge, MA: MIT Press.

Alesina, A., Carrasquilla, A., y Echavarria, J. J. (2000). Decentralization in Colombia. Working Paper Series. Documentos de Trabajo, (15). Bogotá, Colombia: Fedesarrollo.

Arenas, J. (1985). Cese el Fuego: Una Historia Política de las FARC. Bogotá, Colombia: Editorial Oveja Negra.

Bird, R. M. (1970). Taxation and Development. Lessons from Colombian Experience. Cambridge, MA: Harvard University Press.

Bird, Richard M. (2012). Fiscal Decentralization in Colombia: A Work (Still) in Progress ICEPP Working Papers. 79. Recuperado de https://scholarworks.gsu. edu/icepp/79.

Bottia, M., Cardona Sosa, L., y Medina, C. (2012). El SISBEN como mecanismo de focalización individual del régimen subsidiado en salud en Colombia: ventajas y limitaciones. Revista de Economía del Rosario, 15(2), 137-177.

Cadena, X. (2002). La Descentralización Empereza? Efecto de las Transferencias sobre los Ingresos Tributarios Municipales en Colombia. [Manuscrito]. Documentos CEDE. Bogotá, Colombia: Centro de Estudios sobre Desarrollo Económico - CEDE,.

Clavijo, S. (2004). Requisitos para la Estabilidad Fiscal: Un Balance y la Agenda. Ensayos sobre Política Económica, 46 (1) 146-185.

Colombia, República de. (1981). Misión de Finanzas Intergubernamentales. Informe. Bogotá, Colombia: Departamento Nacional de Planeación.

Dillinger, W., y Webb, S. B. (1999). Decentralization and Fiscal Management in Colombia. Policy Research Working Paper, (2122). Washington, DC., USA: World Bank.

Echavarria, J. J., Rentería, C., y Steiner, R. (2002). Decentralization and Bailouts in Colombia. Research Network Working Papers, (R-442). Washington, D.C., USA: Inter-American Development Bank,

Echeverry, J. C., Fergusson, L., y Querubín, P. (2004). La Batalla Política por el Presupuesto de la Nación: Inflexibilidades o Supervivencia Fiscal. Documento CEDE, (2004-01). Bogotá, Colombia: Centro de Estudios sobre Desarrollo Económico - CEDE.

ECLAC, Economic Commission for Latin America and the Caribbean. (2019). Overall Fiscal Balance. CEPALSTAT. Recuperado de: https://cepalstatprod.cepal. org/cepalstat/tabulador/Consultalntegrada.asp? idlndicador=1246\&idio$\mathrm{ma}=\mathrm{i}$

Gargarella, R. (2015). El "Nuevo Constitucionalismo Latinomericano". Estudios Sociales, 48(1), 169-174. doi:10.14409/es.v48il.5105. 
Ginsburg, T., Elkins, Z., y Blount, J. (2009). Does the Process of Constitution-Making Matter? Annual Review of Law and Social Science, 5(1), 201-223. doi:10.1146/ annurev.lawsocsci.4.110707.172247

Hurtado, M. (2006). Proceso de Reforma Constitucional y Resolución de Conflictos en Colombia: el Frente Nacional de 1957 y la Constituyente de 1991. Revista de Estudios Sociales, (23), 97-104. doi:10.7440/res23.2006.08

Iregui, A. M., Ramos, J., y Saavedra, L. A. (2001). Análisis de la Descentralización Fiscal en Colombia. Borradores de Economia, (175). Bogotá, Colombia: Banco de la República.

Jaimes, R. (2020). Fiscal Adjustments at the Local Level: Evidence from Colombia. International Tax and Public Finance, 27(5), 1148-1173. doi:10.1007/s10797020-09598-2

Jimeno, M. (2001). Violence and Social Life in Colombia. Critique of Anthropology, 21 (3), 221-246. doi:10.1177/0308275X0102100302

Jordana, J., y Gomà, R. (2004). Introducción: Democracia, Descentralización y Política Social. En R. Goma y J. Jordana (Eds.), Descentralización y Políticas Sociales en América Latina (pp. 11-26). Barcelona, España: Fundació CIDOB.

Junguito, R., Melo, L. A., y Misas, M. A. (1995). Descentralización y Finanzas Públicas: Colombia 1967-1994. Ensayos sobre Política Económica, 27, 7-38.

Junguito, R., y Rincón, H. (2004). La Política Fiscal en el Siglo XX en Colombia. Borradores de Economia, (318). Bogotá, Colombia: Banco de la República.

Lassalle, F. (1946). ¿ Qué es una Constitución? Buenos Aires, Argentina: Siglo Veinte Editores.

Lozano, L. I. (2001). Colombia's Public Finance in the 1990s: A Decade of Reforms, Fiscal Imbalance, and Debt. Borradores de Economía, (174). Bogotá, Colombia: Banco de la República.

Lozano, L. I., y Aristizábal, C. (2003). Déficit Público y Desempeño Económico en la Década del Noventa. El Caso Colombiano. Desarrollo y Sociedad(52), 67-97. doi:10.13043/dys.52.3

Mardones Z, R. (2006). Descentralización y Transición en Chile. Revista de ciencia política (Santiago), 26(1), 3-24. doi:10.4067/S0718-090X2006000100001

Melo, L. A. (2005). Impacto de la Descentralización Fiscal sobre la Educación Pública Colombiana. Borradores de Economía, (350). Bogotá, Colombia: Banco de la República.

Musgrave, R. (1959). The Theory of Public Finance: A Study in Public Economy. New York, USA: McGraw-Hill.

Negretto, G. L. (2013). Making Constitutions: Presidents, Parties, and Institutional Choice in Latin America. New York, USA: Cambridge University Press.

O'Donnell, G. A. (1993). On the State, Democratization and Some Conceptual Problems: A Latin American View with Glances at Some Postcommunist Countries. World Development, 21 (8), 1355-1369. doi:10.1016/0305-750X(93)90048-E

Oates, W. E. (1972). Fiscal Federalism. New York, USA: Harcourt Brace Jovanovich.

Oates, W. E. (2005). Toward A Second-Generation Theory of Fiscal Federalism. International Tax and Public Finance, 12(4), 349-373. doi:10.1007/s10797-0051619-9

Perry, G., y Rodríguez, J. A. (1991). Las Finanzas Intergubernamentales en la Constitución de 1991. Coyuntura Económica: Investigación Económica y Social, $21(4), 65-83$.

Prud'Homme, R., y Shah, A. (2004). Centralização versus Descentralização: O Diabo está nos Detalhes (P. M. Soares, Trad.). In F. Rezende y F. A. d. Oliveira (Eds.), Federalismo e Integração Econômica Regional. Desafios para o Mercosul (pp. 63-95). Rio de Janeiro, Brasil: Konrad Adenauer Stiftung.

Restrepo, D. (2002). Luchas por el Control Territorial en Colombia. Economia, Sociedad y Territorio, 3(12), 517-537. doi:10.22136/est002002335

Restrepo, D. (2004). De la Descentralización a la Regionalización. Nuevo Escenario de la Guerra y Oportunidad para la Paz. Revista Eure, 29(89), 81-96. doi:10.4067/SO250-71612004008900005

Restrepo, G., y Alvarez, D. (2005). Ley 617 de 2000 y su Impacto Fiscal Territorial. [Manuscrito]. Ensayos Sobre Economia Regional. Medellín, Colombia: Banco de la República

Rezende, F., y Oliveira, F. A. d. (Eds.). (2003). Descentralização e Federalismo Fiscal no Brasil. Desafios da Reforma Tributária. Rio de Janeiro, Brasil: Fundação 
Konrad Adenaver.

Rezende, F., y Oliveira, F. A. d. (Eds.). (2004). Federalismo e Integração Econômica Regional. Desafios para o Mercosul. Rio de Janeiro, Brasil: Fundação Konrad Adenaver.

Rincón, H., Berthel, J., y Gómez, M. (2003). Balance Fiscal Estructural y Cíclico del Gobierno Nacional Central de Colombia, 1980-2002. Ensayos sobre Política Económica, (44), 12-62.

Rodríguez, J. A. (2003). El Estado Unitario Descentralizado: Una Crítica al Enfoque Constitucional Colombiano. Documento CEDE, (15). Bogotá, Colombia: Centro de Estudios sobre Desarrollo Económico - CEDE

Rodríguez-Takeuchi, L. K. (2009). Los municipios colombianos y el conflicto armado. Una mirada a los efectos sobre la efectividad en el desempeño de los gobiernos locales. Colombia Internacional, (70), 93-120. doi:10.7440/colombiaint70.2009.04

Rubio, M. (2002). Conflicto y Finanzas Públicas Municipales en Colombia. [Manuscrito]. Documento CEDE, (17). Bogotá, Colombia: Centro de Estudios sobre Desarrollo Económico - CEDE

Sánchez, F. (2006). Descentralización y Progreso en el Acceso a los Servicios Sociales de Educación, Salud y Agua y Alcantarillado. Bogotá, Colombia: Departamento Nacional de Planeación.

Sánchez, F., España, I., y Zenteno, J. (2015). Fiscal Cumbia: Colombia's Revenue Dynamism. En V. F. Cibils y T. Ter-Minassian (Eds.), Decentralizing Revenue in Latin America. Why and How (pp. 155-197). Washington DC, USA: Inter-American Development Bank.

Sánchez, F., Olivera, M., y Cortes, G. (1997). Ahorro, Inversión y Transferencias en las Entidades Territoriales Colombianas. Archivos de Macroeconomía (67). Bogotá, Colombia: Departamento Nacional de Planeación

Sánchez, F., Smart, M., y Zapata, J. G. (2002). Intergovernmental Transfers and Municipal Finance in Colombia. Working Paper Series. Documentos de Trabajo, (2915). Bogotá, Colombia: Fedesarrollo

Théret, B. (2015). La Diversidad de los Federalismos en América Latina: Argentina, Brasil y México. En I. Bizberg (Ed.), Variedades del Capitalismo en América Latina: Ios Casos de México, Brasil, Argentina y Chile (pp. 147-281). México DF, México: El Colegio de México.

Trejos Rosero, L. F., Guzmán, J., Badillo Sarmiento, R., y Martínez Martínez, F. (2020). Clientelismo armado en Barranquilla: el caso del Frente José Pablo Díaz (20032006). Justicia, 25(37), 49-70. doi:10.17081/just.25.37.4129

Uribe, D. (1985). Las Constituciones de Colombia (segunda ed.). Madrid, España: Instituto de Cooperación Iberoamericana.

Weingast, B. R. (2009). Second Generation Fiscal Federalism: The Implications of Fiscal Incentives. Journal of Urban Economics, 65(3), 279-293. doi:10.1016/j. jue.2008.12.005

Weingast, B. R. (2014). Second Generation Fiscal Federalism: Political Aspects of Decentralization and Economic Development. World Development, 53, 14 25. doi:10.1016/j.worlddev.2013.01.003

Wiesner, R. (2004). El Origen Político del Déficit Fiscal en Colombia: El Contexto Institucional 20 años Después. Documento CEDE, (20). Bogotá, Colombia: Centro de Estudios sobre Desarrollo Económico - CEDE 